Measures included: start time, length of handover, number of bleep interruptions, time of specialty handover, and missed patients.

Problems identified included:

- Late specialty handovers resulting in delays to commencing shifts

- Frequent non-urgent bleeps interrupting flow and causing distraction

- Patients not handed over in order meaning patients 'missed'

- Multiple late 'corridor handovers'

These findings were presented to different stakeholders to address concerns, identify good practice and suggest areas for improvement. Suggestions included; introduction of a handover checklist, reminders on ward phones of protected handover times, and the introduction of a traffic light system to classify patients into an acuity category.

Intervention Utilising small tests of change (PDSA cycles) we implemented sequential changes. For example a handover checklist was introduced at the start of each handover. Simple additions such as shutting the door gave clear non-verbal clues to people who were late. Stratifying patients according to traffic light acuity red (un-stable) amber (potentially unstable) and green (stable and on pathway) helped keep handovers focused.

Strategy for change These changes were introduced and measured over an additional 16 handovers with continual feedback from junior doctors and outreach nurses. The consultant body was kept informed by the project lead.

Measurement of improvement Measurements were undertaken over specific periods rather than continually. The project began in the quieter summer months with lower total patient numbers and the $3^{\text {rd }}$ data collection is from November during busier times on the unit.

Effects of changes Several small changes have considerably improved the handover process. Verbal feedback has been really positive. Some of the initial changes have now been incorporated into the trust-wide computer system and are now used routinely in adult practice.

We are continuing to measure variables and make changes. Uptake of some of the changes is variable (see results, Table 1), however as we reinforce successful changes and introduce handover education into induction, we hope to make adjustments standard procedure.

Next steps are to develop a standardised structure for handover of patients according to acuity category. This will be linked to need for review and outreach involvement.

Lessons learnt Getting buy-in from the consultant body and key stakeholders has been paramount. The senior lead empowering the juniors who are present on a daily basis to continue change when faced with those less willing to engage in a new process has been essential to success.

Using regular feedback has enabled us to review improvements. We have been able to re-adjust changes on a short time scale, enabling us to reflect on the impact of our adjustments on the safety of handover.

Message for others Simple rapid small changes have significantly improved the efficiency and safety of handover. Front line junior staff, empowered by more senior clinicians, have introduced these changes. Engaging the whole team is critical to sustained success. Change is a continual dynamic process and monitoring effects of interventions helps identify where further work is needed.

\section{G532(P) THE CARE NEVER STOPS: IMPROVING CHILD DEATH MANAGEMENT}

CS Sen, I Heyes, P Outram. Paediatrics, Ealing Hospital, London, UK

10.1136/archdischild-2015-308599.483

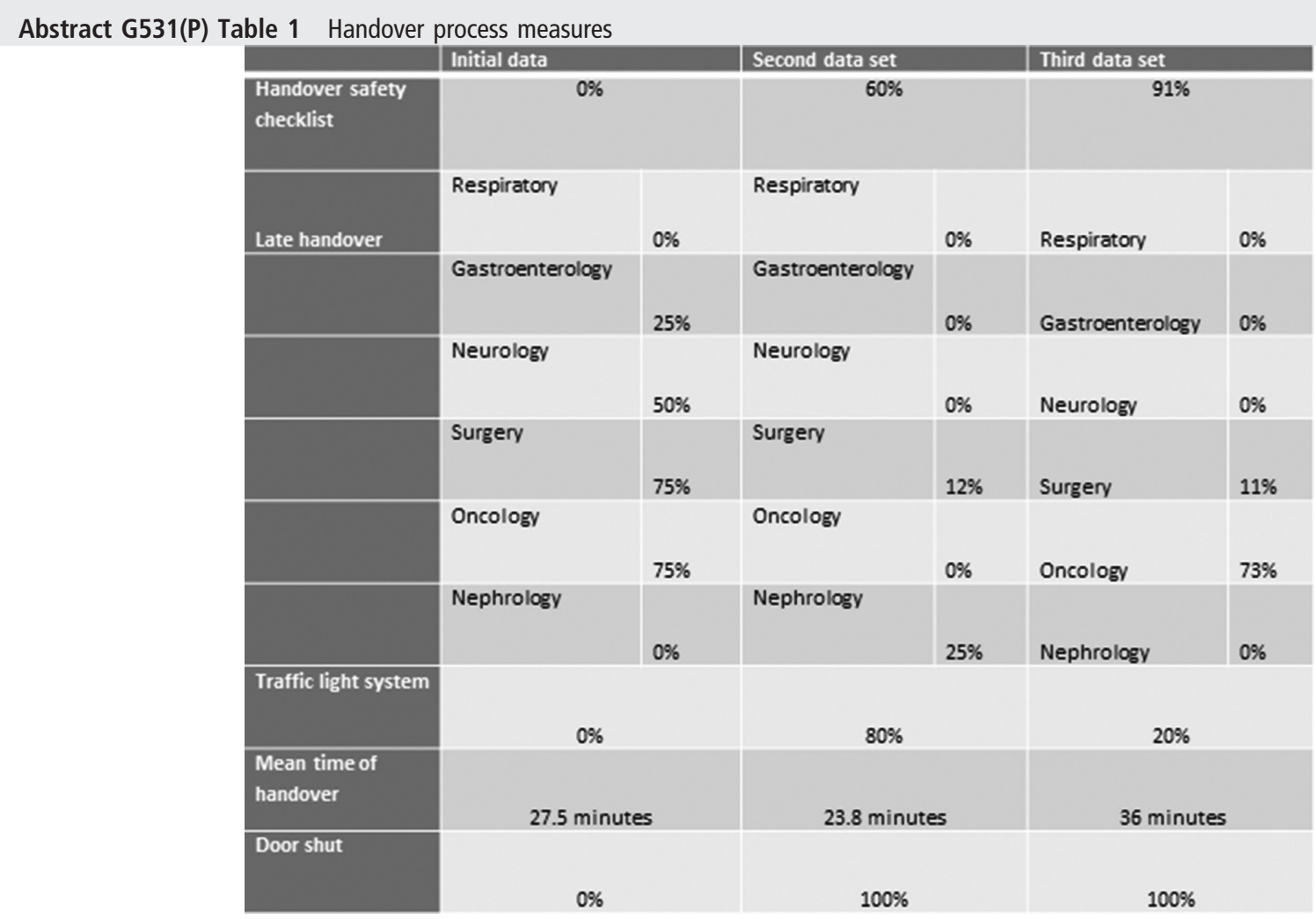


Context Every year almost 3000 children and young people die between the ages of 1 and 19 (ChildBereavementUK). The care that a child receives from medical professionals around the time of their death has lifelong implications for the family.

Problem At our hospital $70 \%$ of paediatric staff surveyed were not confident to offer support to bereaved families.

Assessment of problem and analysis of its causes The current bereavement leaflet was out of date and only relevant to the death of an adult. The staff surveyed felt they had not had adequate training.

Interventions

- Development of an updated Paediatric Bereavement Support Pack containing relevant information to support bereaved families and guidelines for staff on completion of legal formalities

- Training to increase the confidence of paediatric doctors and nurses in supporting bereaved families

- Training to improve the confidence and understanding of the procedures required after a child has died

Study design We surveyed 22 doctors and nurses working across the paediatric department including the ward, the paediatric emergency department and the special care baby unit. We asked them whether they were aware of the current bereavement pack, how to access it and whether they knew what support was available to grieving families. We asked them to identify agencies and professionals who should be informed of the death of a child.

Strategy for change We used a focus group to guide our development of a new leaflet appropriate for the death of a child and sensitive to a multicultural society. This highlighted that a checklist would be useful. The checklist included legal documentation required, professionals and agencies to inform, and references bereavement support references. We created bereavement packs containing all essential forms, the checklist and the updated bereavement leaflet and placed them in each of the main paediatric hospital departments. We conducted several multidisciplinary teaching sessions involving the named consultant for child death, the named nurse for safeguarding and a representative from the Child Death Overview Panel. They aimed to demystify the processes surrounding child death and to increase people's confidence in dealing with the death of a child.

Measurement of improvement Following our interventions and training we used the same questions to reassess the knowledge and confidence of 22 paediatric doctors and nurses.

Effects of changes Awareness of the bereavement pack increased from $55 \%(12)$ to $100 \%(22)$. Awareness of support available to families increased from $36 \%(8)$ to $95 \%(21)$. Before our interventions $55 \%(12)$ people surveyed were aware of the name of the named doctor for child death and afterwards 86\%(19). Only $40 \%(9)$ of people surveyed before our interventions were aware of more than 3 agencies that needed to be informed of the death of a child; this rose to $82 \%(18)$ after the interventions. All of the agencies are listed on the checklist included in the pack. Overall the confidence level of staff to support newly bereaved families improved from 23\%(5) to 55\%(12) (Figure 1). Feedback from the teaching described it as "informative", "interactive" and "a clear pathway".

Lessons learnt This study demonstrated the lack of confidence and knowledge of paediatric staff in management of child death and support of bereaved families. Our training and interventions improved confidence in dealing with child death.

Message for others Consultation with nursing and medical staff involved in child death is important in ensuring useful checklists and training. However, as child death is an infrequent and stressful event regular teaching is required to maintain and strengthen confidence.

\section{G533(P) DEVELOPMENT AND EVALUATION OF STANDARDISED FOLLOW UP PATHWAY FOR HENOCH SCHOENLEIN PURPURA}

H Unsworth, H Massoud. General Paediatric Department, The Royal Wolverhampton Hospital NHS Trust, Wolverhampton, UK

\subsection{6/archdischild-2015-308599.484}

Context The quality improvement involved development of a follow up service for patients presenting to a busy district general hospital with Henoch Schoelein Purpura (HSP). Follow up is recommended as the rare but important renal sequelae of the condition can be silent initially.

Problem In our department no follow up pathway existed for HSP. Patients were seen in either consultant outpatient clinics, with the majority of children being well, or discharged for primary care follow up which relies on general practitioners and families to organise ill defined follow up creating the potential to miss any renal sequelae

Assessment of problem and analysis of its causes The problem was identified during the acute assessment of patients presenting. Following discussion with senior staff about absence of evidence based local follow up procedures and subsequent literature review a structured pathway was proposed. It was felt that all children should have hospital review with consultant follow up focusing on children with complications

Intervention The aim was to standardise and rationalise care thereby improving department efficiency without compromising patient safety. A pathway for nurse led follow up which had been piloted elsewhere (1) was used as a model. Children were assessed at presentation and guidance was given for those needing admission or investigation. Otherwise they should be discharged home with urine dipsticks to check early morning urine and information about what concerning features should lead them to seek

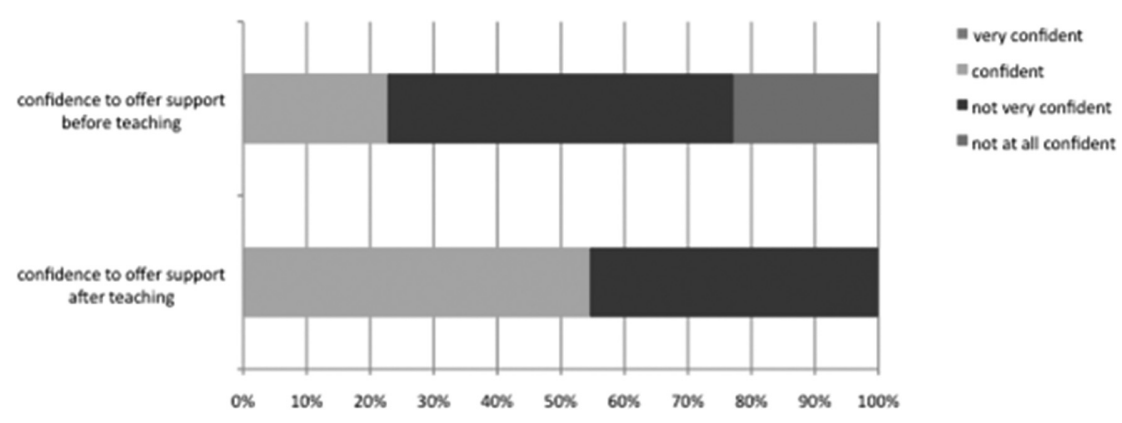

